\title{
EDITORIAL
}

\section{Lectura crítica de la literatura médica: a propósito de la relación entre la terapia de reemplazo hormonal y el riesgo de cáncer de seno}

\author{
Enrique Ardila Ardila MD*; Ariel Iván Ruiz Parra MD**
}

El médico se ve enfrentado cada vez más a un gran volumen de publicaciones. Esta información debe ser previamente seleccionada y evaluada con el objeto de mejorar la comprensión y aplicación a la práctica diaria. Este fue el motivo por el cual se desarrolló la metodología de la "lectura crítica" (1-2).

Los dos modelos de "lectura crítica" más aceptados internacionalmente son los propuestos por los grupos de Epidemiología Clínica de las Universidades de McMaster en Canadá (3) y New Castle en Australia (2). Estos dos sistemas comparten una metodología similar y pretenden situar al lector de una forma crítica y analítica frente al diseño empleado por los autores, a sus resultados $\mathrm{y}$ conclusiones.

Según la metodología de la universidad de McMaster (3) los puntos fundamentales para tener en cuenta en la lectura son:

1. Título del artículo

2. Autores y lugar donde laboran los investigadores

3. Resumen

4. Lugar en donde se llevó a cabo la investigación

5. Interés específico del lector

De acuerdo con la metodología canadiense, la importancia de cada uno de estos puntos radica en los siguientes aspectos:

El título del artículo le suministra al lector una idea general sobre el interés y la utilidad de la investigación. En el caso que nos ocupa no hay ninguna duda de que el momento actual reviste una importancia capital el artículo de Colditz GA y colaboradores acerca del uso de estrógenos y progestágenos y el riesgo de cáncer de seno en mujeres posmenopáusicas (4).

Un lector experto en un área determinada del conocimiento reconoce en forma rápida la trayectoria de muchos investigadores y los autores del artículo le brindan

Profesor Asociado de Medicina Interna y Endocrinología.

Universidad Nacional de Colombia

Director de Recursos Humanos

Ministerio de Salud

Profesor Asociado de Ginecología y Obstetricia

Universidad Nacional de Colombia

Coordinador Unidad de Endocrinología Ginecológica

Instituto Materno Infantil información sobre la seriedad e importancia de la investigación. No obstante, los lectores no tienen que ser siempre profundos conocedores de todos los temas y además los investigadores nuevos no siempre tienen que ser conocidos. En estas circunstancias el lugar donde laboran los investigadores proporciona información adicional importante. En esta publicación del New England Journal of Medicine, tanto los autores como el sitio en donde trabajan (Escuela de Medicina de la Universidad de Harvard) son ampliamente conocidos.

Al revisar el resumen de un artículo el lector puede evaluar si los resultados, cuando son válidos, pueden ser útiles y se justificaría la lectura en extenso del artículo.

El lugar en donde se llevó a cabo la investigación reviste importancia en relación con las áreas epidemiológica y demográfica por cuanto le permite al lector decidir sobre la aplicabilidad de los resultados a su propia práctica. Sobre el tema tratado en el artículo de Colditz puede existir un sesgo de selección ya que se trata de una muestra limitada a un grupo de mujeres profesionales de la salud.

Los intereses más comunes por los cuales los médicos consultan la literatura médica y los puntos que se deben valorar en la sección de "pacientes y métodos" en cada caso particular son los siguientes (3):

1. Para evaluar una prueba diagnóstica:

- ¿Se incluyó en la muestra de pacientes un espectro apropiado de la enfermedad?

- ¿Se hizo una comparación independiente y a ciegas con un "estándar de oro?

- ¿Se determinó la reproducibilidad de la prueba y la variación del observador?

- ¿Se describió el medio dónde se desarrolló el estudio y el patrón de referencia de los pacientes?

- ¿Se definió adecuadamente el término "normal"?

- Si la prueba forma parte de un grupo de pruebas, ¿se analizó su contribución a la validez total?

- ¿Se describió la prueba de tal forma que permita su replicación exacta?

- ¿Se determinó la "utilidad" de la prueba?

2. Para conocer el curso y pronóstico de una enfermedad:

- ¿Se describió el patrón de referencia de los pacientes? 
- ¿Se obtuvo un seguimiento completo?

- ¿Se utilizaron criterios objetivos para evaluar los resultados?

- ¿Los resultados se analizaron en forma ciega? ¿Se hicieron ajustes para otros factores pronósticos?

3. Para encontrar la etiología de una enfermedad:

- ¿Hay evidencia de experimentos verídicos en humanos?

- ¿La asociación es fuerte?

- ¿La asociación es consistente en todos los estudios?

- ¿Hay una correcta relación temporal?

- ¿Hay un gradiente dosis-respuesta?

- ¿Tiene sentido epidemiológico la asociación?

- ¿La asociación tiene sentido desde el punto de vista biológico?

- ¿La asociación es específica?

- ¿La asociación es análoga a una asociación causal demostrada previamente?

4. Para evaluar los riesgos y beneficios de un tratamiento:

- ¿Se informaron todos los eventos clínicos relevantes?

- ¿Los pacientes estudiados son similares a los que el lector asiste?

- ¿Se consideraron la significancia estadística y la significancia clínica?

- ¿El tratamiento es factible en su práctica clínica?

- ¿Se obtuvo un seguimiento completo?

Usando la metodología propuesta por la universidad de New Castle (2) se puede utilizar una "rejilla" que resume los aspectos básicos de la "lectura crítica" como son: objetivo, tipo de estudio llevado a cabo, factores estudiados, criterios de juicio, origen de la población y sujetos estudiados, factores que podrían potencialmente producir confusión en el estudio, análisis estadístico y conclusiones. Como se puede deducir estos dos métodos son complementarios y es recomendable tenerlos en cuenta en forma simultánea en el momento de realizar nuestra "lectura crítica".

En nuestro caso el artículo se refiere al riesgo de padecer cáncer de seno cuando se utiliza terapia de reemplazo hormonal. El diseño de la investigación se llevó a cabo siguiendo el modelo metodológico de "casos y controles" el cual es aceptado como válido para responder a este tipo de interrogantes.

En el artículo publicado por Colditz y cols. los resultados se obtuvieron del seguimiento de 725.550 mujeres-año, en quienes se detectaron 1.935 casos de carcinoma invasivo del seno en mujeres posmenopáusicas. El riesgo relativo de padecer cáncer de seno en relación con la exposición a varios factores, especialmente a la terapia hormonal sustitutiva de estas pacientes, se analizó en forma estratificada, buscando la importancia específica que pudiera tener la exposición a estrógenos, estrógenos mas progestágenos, progestágenos solos y estrógenos más testosterona.

El riesgo relativo (RR; relación entre la incidencia de la enfermedad entre expuestos/no expuestos) de cáncer de seno en las mujeres expuestas a estrógenos conjugados solos fue de 1.32 (IC 95\%: 1.14-1.54), el RR en las mujeres expuestas a estrógenos más progestágenos fue de 1.41 (IC 95\%: 1.15-1.74) y en las mujeres expuestas a progestágenos solos el RR fue de 2.24 (IC 95\%: 1.263.98). Esto significa que las mujeres posmenopáusicas que utilizan estrógenos tendrían mayor riesgo de padecer cáncer de seno, que quienes no utilizan esta terapia y, por otro lado, la terapia combinada con progestágenos no modifica en ningún sentido este aumento del riesgo.

Los autores también encontraron que el aumento significativo del RR de padecer cáncer de seno ocurría solamente cuando las mujeres continuaban utilizando la terapia hormonal de reemplazo y la habían utilizado por más de cinco años y que el RR de muerte por carcinoma de seno en la población expuesta a esta terapia fue de 1.45 (IC 95\%: 1.01-2.05).

No obstante, el RR de mortalidad total ajustado para historia familiar e historia de enfermedad benigna del seno fue de 1.14 (IC 95\%: 0.85-1.51) para las mujeres que en el momento continuaban usando las hormonas y de 0.8 (IC 95\%: 0.60-1.07) para quienes las habían usado anteriormente. Esto significa que la mortalidad total no es superior a la de la población no expuesta a las hormonas.

Metodológicamente esta publicación está bien llevada a cabo; aunque no conocemos las dosis utilizadas de las diferentes hormonas, sus resultados son válidos en la población estudiada. Debe llamarse la atención que los resultados mostraron un aumento del riesgo de muerte solamente por cáncer del seno. No ocurrió así con el riesgo de morir por otras causas.

De hecho, se considera que el riesgo que tienen las mujeres posmenopáusicas mayores de 50 años de morir por causas selecionadas es (5):

Enfermedad coronaria

Fractura de cadera

$31.0 \%$

Cáncer del seno

$2.8 \%$

Cáncer endometrial

$2.8 \%$

$0.7 \%$

Estos datos nos muestran que el riesgo de morir por otras causas supera ampliamente al del cáncer del seno. De otro lado, los beneficios encontrados en las mujeres posmenopáusicas que reciben terapia hormonal de reemplazo han sido ampliamente demostrados. Estos incluyen una reducción del 50\% del riesgo de padecer y de morir de una enfermedad cardiovascular (6), una reducción similar del riesgo de sufrir una fractura ocasionada por osteoporosis y una mejor calidad de vida, además de una disminución de un $40 \%$ en la mortalidad por todas las causas en las mujeres que recibieron estrógenos por mas de quince años (7).

Estas cifras indican claramente que la relación riesgo / beneficio se inclina a favor de la utilización de la terapia hormonal de reemplazo. Sin embargo, el médico tratante deberá de prestar particular atención a los factores de riesgo para carcinoma de seno y al seguimiento estricto de las pacientes que reciben estrógenos, así como a las pocas contraindicaciones absolutas de la terapia hormonal.

El esquema óptimo es la terapia con estrógenos solos en las pacientes histerectomizadas y la terapia combinada con progestágenos en las mujeres a quienes no se les ha practicado tal intervención (8).

El lector deberá estar atento a las publicaciones sobre el tema que irán apareciendo y tener siempre en cuenta la metodología de "lectura crítica" de la literatura, con el fin de aplicar estos datos al manejo de sus pacientes. 


\section{BIBLIOGRAFIA}

1. Sackett DL., Haynes RB., Tugwell P. Clinical Epidemiology. A Basic Science for Clinical Medicine. First Ed. Boston: Little, Brown and Comp. 1985.

2. Landrivon G., Delahaye F., Ecochard R., Colin C., Heller RF., Ardila E. Principios de lectura crítica de la literatura médica - relación con los diferentes modelos de investigación clínica. Médicas UIS 1993; 2(2): 95-100.

3. Department of Clinical Epidemiology and Biostatistics, McMaster University Health Science Centre. Clinical Epidemiology Rounds: How to read clinical journals. I - V. Can Med Assoc J 1981; 124: 555 558, 703-710, 869-872, 985-990, 1156-1162.

4. Colditz GA., Hankinson SE., Hunter DJ., Willett WC., Manson JE., Stampfer MJ et al. The use of estrogens and progestins and the risk of breast cancer in postmenopausal women. N. Engl. J. Med. 1995; 332(24): 1589-1593.
5. Stumpf PG., Trolice MP. Compliance problems with hormone replacement therapy.Obstet.Gynecol. Clin. North .Am.1994; 21(2): 219-229.

6. StampferMJ., Colditz TA., Willett WC et al. Postmenopausal estrogen therapy and cardiovascular disease - ten year follow up from the Nurses' Health Study. N. Engl. J Med. 1991; 325: 756-762.

7. Henderson BE., Paganini-Hill A., Ross RK. Decreased mortality in users of estrogen replacement therapy. Arch. Intern. Med. 1991; 151: 75-78.

8. Davidson NE. Hormone replacement therapy - Breast versus heart versus bone. N. Engl. J. Med. 1995; 332(24): 1638-1639. 